\title{
A neurocomputational model of stochastic resonance and aging
}

\author{
Shu-Chen $\mathrm{Li}^{\mathrm{a}}{ }^{*}$, Timo von Oertzen ${ }^{\mathrm{b}}$, Ulman Lindenberger ${ }^{\mathrm{a}}$ \\ ${ }^{a}$ Center for Lifespan Psychology, Max Planck Institute for Human Development, Lentzeallee 94 D-14195 Berlin, Germany \\ ${ }^{\mathrm{b}}$ Department of Mathematics, Saarland University, D-66123 Saarbrücken, Germany
}

Received 3 August 2004; received in revised form 8 April 2005; accepted 16 June 2005

Available online 14 November 2005

Communicated by T. Heskes

\begin{abstract}
Stochastic resonance (SR) is fundamental to physical and biological processes. Here, we use a stochastic gain-tuning model to investigate interactions between aging-related increase of endogenous neuronal noise and external input noise in affecting SR. Compared to networks that have optimal system gain parameter of the activation function, networks with attenuated endogenous gain tuning at the system level, simulating aging neurocognitive systems with more intrinsic neuronal noise but less plasticity, continue to exhibit the general SR effect; however, this effect is smaller and requires more external noise. This set of finding suggests that determining the optimal proportion of resonance-inducing external noise as a function of internal-system stochastic gain tuning properties promotes unified theorizing about sensory and cognitive aging at behavioral and neural levels of analysis.
\end{abstract}

(C) 2005 Elsevier B.V. All rights reserved.

Keywords: Stochastic resonance; Neuromodulation; Brain aging; Neural network; Sensory detection

\section{Introduction}

Stochastic resonance (SR), the phenomenon of noiseenhanced responses to weak signals, is fundamental in many physical as well as physiological processes $[10,44]$. For instance, recent evidence of SR in neurobiological systems' sensory processing suggests that SR increases phase locking and coherence, thus promoting synchronization of neuronal activities (for a review, see [29]). Specifically, in humans, an optimal level of noise externally added to subthreshold signals can improve tactile sensory detection [6,7,25], balance control [12,32], and visual perception [38]. A recent fMRI study [39] also showed that SR enhanced the activation level of neuronal activity in human visual cortex.

Other than external noise, intrinsic stochasticity of the nervous system is also inherently present in the components of neurons, such as synapses, that are central for

\footnotetext{
*Corresponding author. Tel.: + 493082406305 ; fax: + 49308249939 .

E-mail addresses: shuchen@mpib-berlin.mpg.de (S.-C. Li), timovoe@gmx.de (T. von Oertzen), seklindenberger@mpib02.mpib-berlin. mpg.de (U. Lindenberger).
}

neurotransmission [17]. Sensory and cognitive processes entail constant exchanges between neuronal activities and external stimulations. Thus, interactions between internal and external noise are inevitable. In order to study such interactions, it has been suggested recently that computational approaches need to explore how between-system differences in endogenous system parameters that regulate internal stochasticity interact with the commonly observed SR that is induced by external noise [e.g., 14,46]. With respect to gerontological applications, it has been suggested that senescence is associated with increasing internal noise. For instance, a classical hypothesis in neurocognitive aging research states that the aging brain is noisier [42], due to deteriorations in various transmitter systems $[1,2,13,15$, $35,36,45]$, such as acetylcholine (Ach) and monoamines (e.g., dopamine, norepinephrine, and serotonin), and degenerations in structural integrity, such as reductions in gray and white matter density and brain volume shrinkages in prefrontal cortex and hippocampus [4,33] as well as attenuated functional connectivity between these cortical regions [11]. Brain electrophysiological activities captured by EEG recordings show aging-related increase in variability [16]. Noisy fluctuations in cognitive and sensorimotor 
processes also increase with aging $[19,22,23,27]$. A handful of studies have examined the effect of external input noise on tactile sensation or balance control in young and old adults $[6,7,12,25,32]$, and provided first evidence of the continued existence of external-noise-enhanced SR in aging neurocognitive systems. However, little is known about how interactions between external input noise and agingrelated increase of endogenous neuronal noise may modulate the general SR effect.

\subsection{Modeling neuromodulation as stochastic system gain tuning}

Tuning the gain parameter of neural networks' activation function is a common system-based approach for modeling neuromodulation and its effect on neural plasticity $[8,37]$. We extended a stochastic gain-tuning model of neurocognitive aging [18] to investigate interactions between external-noise-enhanced SR and agingrelated increase of endogenous neuronal noise derived from deficits in the intrinsic system neuromodulatory process. Rather than assuming additive internal noise $[9,26]$, here we capture aging-related increase of intrinsic neuronal noise that may be attributable to decline in neuromodulation, particularly dopaminergic modulation, by attenuating the gain parameter of neuronal networks' sigmoid activation function (Eq. (1)). The gain parameter is a system parameter that affects the slope and non-linearity of the neural network's activation function. Modeled as such, the net input a given processing unit $i$ receives from afferent channels at the simulated discrete time step $t$ is gated by the gain parameter $\left(G_{i_{t}}\right)$ before the unit emits its activation and further propagates the activity. At each processing step, indexed in discrete time steps, the value of the gain parameter associated with a given unit is randomly sampled from a uniform distribution with a given mean and standard deviation. The stochastic gain manipulation implemented as such incorporates the probabilistic nature of transmitter release [30]:

$\operatorname{Act}\left(G_{i_{t}}\right.$, ,nput $\left._{i_{t}}\right)=\frac{1}{1+\mathrm{e}^{-\left(G_{i_{t}} \text { input }_{i_{t}}+\text { bias }\right)}}$.

Whereas the level of external noise that is part of the stimulus environment may vary leading to various extents of external-noise-induced tuning, systems may also differ in the efficacy of their endogenous gain control mechanism. The stochastic gain manipulation we propose here is aimed at capturing aging-related differences in endogenous neuromodulatory gain control, in order for us to explore the effects of aging on the commonly observed externalnoise-enhanced SR. Differences in neuromodulatory gain control between young and older neurobiological systems can be simulated by neural networks with a larger or smaller mean $G$. Fig. 1 shows three families of activation functions that are associated with three ranges of $G$. The slopes and non-linearity of activation functions associated with smaller $G$ are reduced in comparison to the functions

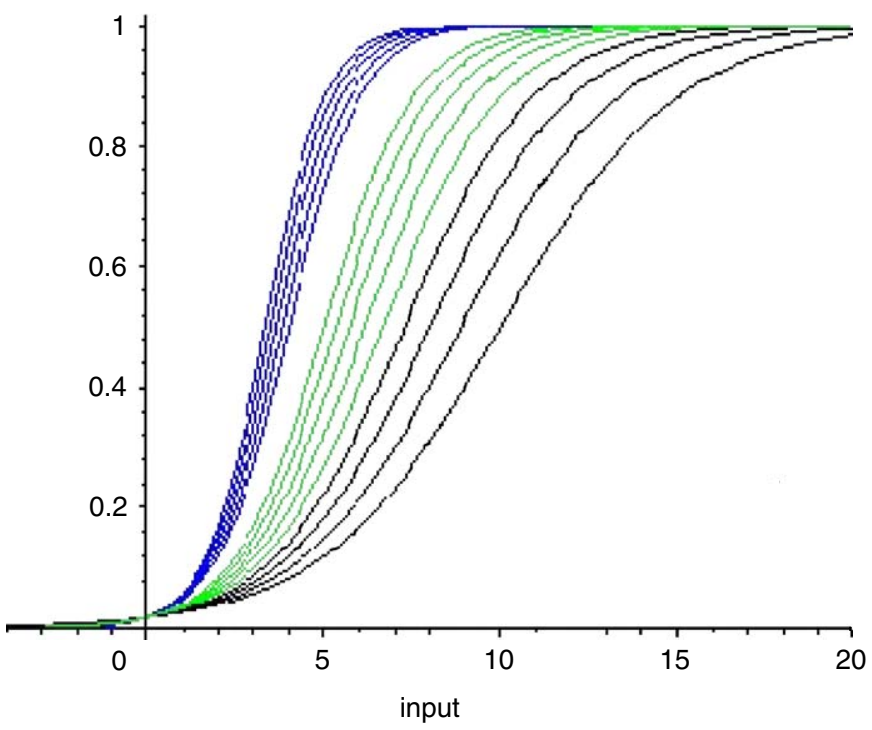

Fig. 1. Three families of sigmoid activation functions. Ranges of the $G$ distributions are [1.0, 1.2], [0.6,0.8], and $[0.4,0.6]$ for the sets of curves on the left, in the middle, and on the right, respectively.

with larger $G$, i.e., comparing the set of curves on the right ( $G$ ranges from 0.4 to 0.6 , with a mean of 0.5 ) and the set of curves in the middle ( $G$ ranges from 0.6 to 0.8 , with a mean of 0.7 ) with that on the left ( $G$ ranges from 1.0 to 1.2 , with a mean of 1.1). Extant evidence suggests that across various brain regions, there is about $5-10 \%$ decline in the efficacy of dopaminergic modulation per decade starting at about age 20 years $[1,15]$. Assuming a $10 \%$ decline per decade, changing the level of mean $G$ from 1.1 to 0.7 numerically corresponds to about four decades $(36 \%)$ of decline (i.e., about the decline from 20 to 60 years of age), whereas changing the level of mean $G$ from 1.1 to 0.5 numerically reflects about six decades $(55 \%)$ of decline (i.e., about the decline from 20 to 80 years of age). These parameter ranges are also comparable to empirically observed aging-related increase in perceptual/cognitive processing fluctuation (noise), which was found to be about $7 \%$ increase in processing noise per decade from age 30 to 90 years in one study [23].

Attenuating the system-parameter-based stochastic gain regulation reduces the responsivity, thus the signal transmission efficiency, of the processing units, which subsequently increases intra-network activation noise and reduces representation distinctiveness [18]. This sequence of effects integrates findings of aging-related decline in neuromodulation $[1,15]$ and various cognitive aging deficits (e.g., adult age differences in learning rate, susceptibility to interference, working memory, associative binding, and performance variability) at the behavioral level [19-22]. However, the question of whether similar mechanisms may also affect SR in aging neurobiological systems still needs to be investigated.

In principle, the within-system manipulation of neural network's gain parameter is related to typical concepts of input or output signal-to-noise ratio (SNR), as it is related 
to the SNR gain frequently encountered in the literatures of perceptual/sensory discrimination or signal processing. However, there is one clear difference: whereas the typically SNR gain [34] is often defined as the ratio of the system's output SNR (e.g., a person's perceptual performance measured at the behavioral level) to input SNR (e.g., the quality of the input stimuli), the withinsystem stochastic gain regulation of the activation function as implemented here is a manipulation of a system parameter that is intrinsic to the neural network. Conceptually, the importance of comparing SR phenomena derived from adding external noise and tuning system parameters as well as studying their interactions have been emphasized in recent work [e.g., 14,46]. With respect to gerontological applications, it is important to arrive at computational frameworks within which between-person differences in endogenous neurobioloical processes, such as the effect of aging on neuromodulation, can be directly modeled by an intrinsic system parameter. If this is done, we are enabled to study how gain regulation of the network's activation function at the system level interacts with the effect of external noise on SR. From an applied perspective, computational and empirical findings on this interaction between external noise and internal gain tuning properties open the possibility for determining optimal levels of noise [cf. 14] for human systems with suboptimal gain tuning characteristics.

Two important earlier studies have shown that external, context-dependent noise linearizes the input-output transfer function of model neuron ensembles [3] and changes the frequency tuning properties of actual sensory neurons [14]. Building on these earlier findings regarding external-noiseinduced tuning of SR, particularly the so-called aperiodic $\mathrm{SR}$, our effort here is to illuminate how external-noiseinduced SR effects vary as a function of between-system

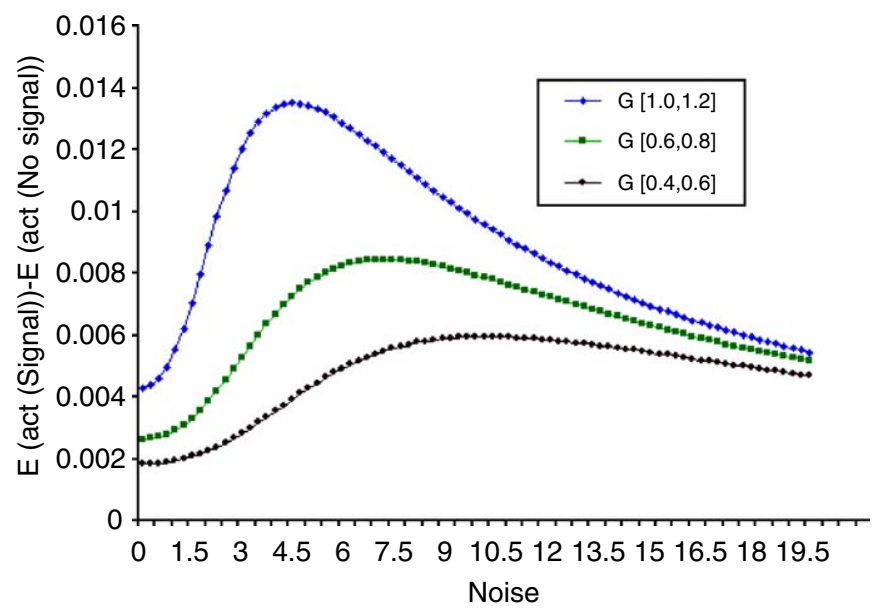

Fig. 2. Detection accuracy as a function of external noise for different levels of $G$ as characterized by the single-unit model. Three families of activation functions with their $G$ values have been drawn from uniform distributions of equal range (i.e., [0.4-0.6], [0.6-0.8], and [1.0-1.2] for the bottom, middle, and top curves, respectively) but differing means. The $y$ intercepts of the curves represent signal detection accuracy without external noise.

tributions with smaller means. Across a range of white Gaussian external input noise, we compute how accurate a single unit can distinguish between two relatively similar signals that represent the presence or absence of a stimulus. For each level of external noise, the single unit's detection accuracy is quantified as the difference between the expectation values of the unit's activation when the signal is present or absent. For a given input, noise level (i.e., here defined as the standard deviation of the Gaussian noise distribution, $x$ ), and range of gain $\left[G_{\min }, G_{\max }\right]$, the expectation value of the unit's activation is computed as the integrals over the respective distributions:

$$
E\left(\text { act }_{G}(\text { input }, \text { noise })\right)=\int_{G_{\min }}^{G_{\max }} \frac{1}{\left(G_{\max }-G_{\min }\right)} \int_{-\infty}^{\infty} \frac{\mathrm{e}^{-x^{2}}}{\sqrt{\pi}} \frac{1}{1+\mathrm{e}^{-(G(\text { input }+ \text { noise } x)+\text { bias })}} \mathrm{d} x \mathrm{~d} G .
$$

differences in the efficacy of endogenous gain regulation. Although external noise may affect the input-output transfer function in both young and old neurobiological systems, mechanisms underlying age differences in brain integrity between the young and old that affect the efficacy of neuromodulation add another layer of influence at the system level, that may, in turn, interact with the effects of external noise tuning.

\section{Analytic analysis of gain tuning and $\mathrm{SR}$ in a single-neuron model}

To analytically characterize the effects of attenuating $G$ on SR, we first considered a single-unit model. The aging deficit of neuromodulation is modeled by sampling $G$ of the processing unit's activation function from uniform dis-
We set the bias to -4.0 , input to 0.0 for "signal absent" and 0.2 for "signal present". Fig. 2 shows that activation functions associated with different levels of $G$ all exhibit the typical pattern of SR: detection accuracy initially increases with increasing input noise, reaches a peak at optimal noise level, and decreases gradually thereafter. However, the peak of SR decreases and shifts rightward with decreasing $G$. These effects show that a unit with smaller $G$ profits less efficiently from SR: the effect of SR is weaker and needs to be achieved with more external noise.

\section{Gain tuning and SR of sensory detection in multi-neuron networks}

We next expanded the $G$ modulation of SR in the singleunit model to multi-unit networks to study adult age 


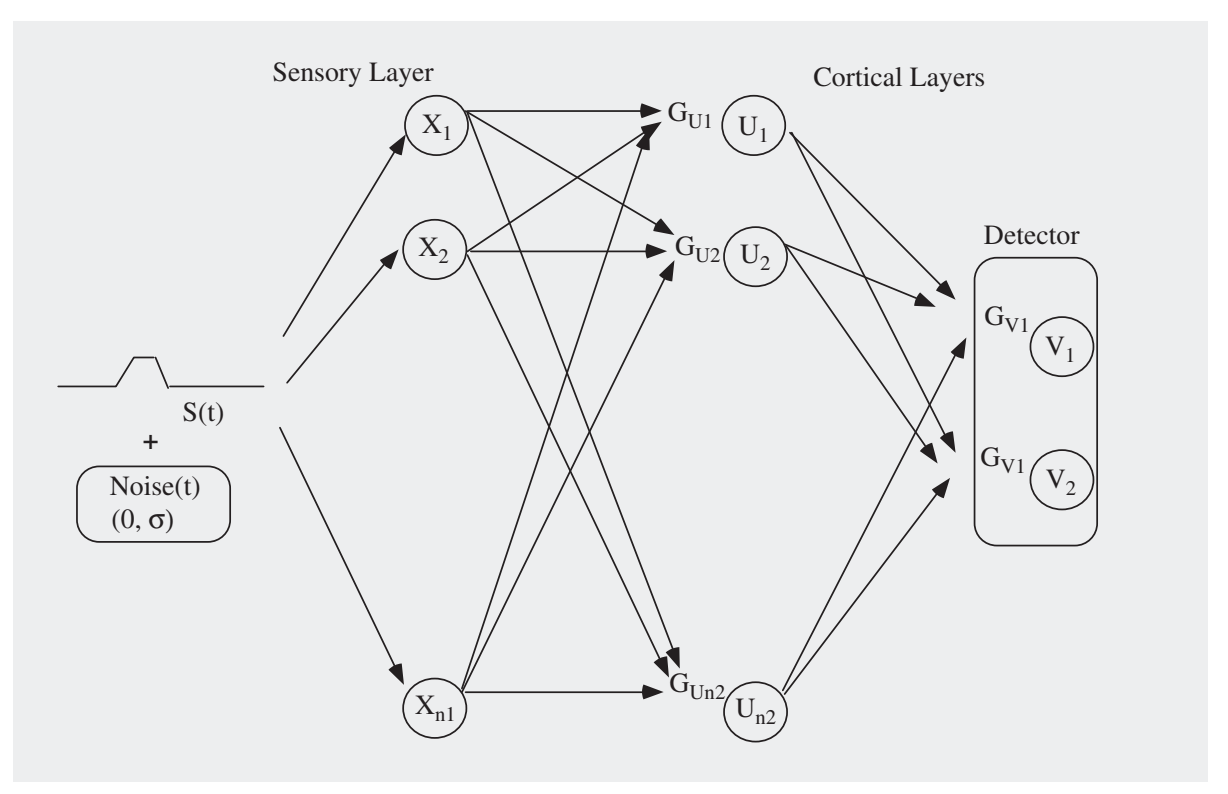

Fig. 3. Schematic diagram of the multi-unit, multi-layer network of sensory detection.

differences in external-noise-enhanced sensory detection. Multi-layer, feedforward backpropagation networks with full connections were implemented (see Fig. 3) to simulate sensory detection in paradigms analogous to earlier experiments [6,7,25]. For all reported simulations, the ensemble of processing units at the sensory layer $\left(n_{1}=12\right)$ encoded a pattern of signal amplitude in arbitrary units that represented the presence (signal value set to 1 ) or absence (signal value set to 0 ) of applied stimulus force across 12 arbitrary discrete time periods. Their activities were summed and the resulting net sensory input to each of the units in the cortical layers $\left(n_{2}=4\right)$ was gated by the units' $G$ parameters. The latter of the two cortical layers had two detector units. Based on activations of the detector, the networks gave a "stimulus" or "no-stimulus" response.

We generated three groups of 10 networks that differed only in the means of the uniform distributions from which the values of their $G$ parameters were sampled. As with the single-unit model, the ranges (hence the standard deviations as well) of the three distributions of $G$ parameters were fixed at 0.2 (i.e., $[0.4,0.6]$, [0.6, 0.8], and $[1.0,1.2]$ ) and the bias of the activation function was fixed at -4.0 . The networks were first trained with the backpropagation algorithm to detect the presence of the perfect stimulus in the absence of external noise until all networks reached an equivalent level of high precision. At testing, the networks were presented with a subthreshold stimulus. The test involved 20 trials (10 "stimulus present" and 10 "stimulus absent") at various levels of external input noise. Independent white Gaussian noise distributions with a mean of zero were added to the sensory signal train. The standard deviations of the noise distributions were varied across blocks. A subthreshold stimulus was generated by reducing the signal intensity of the perfect stimulus to a level that networks with all levels of $G$ achieved the base rate of $50-55 \%$ correct detection accuracy in the absence of any external noise. This criterion was reached by cutting down the signal intensity of the perfect stimulus to $40 \%$ in the reported simulations.

\section{Results}

The results show that the details of SR depend on the interaction between external noise and $G$. In line with SR observed in old people [25], the $G$-reduced networks also exhibit SR. The magnitudes of peak SR are, however, smaller in $G$-reduced networks and the peaks are again right-shifted to greater levels of external noise (Fig. 4(a)). These findings indicate that increased internal noise in the $G$-reduced networks reduces the relative efficiency of external noise in producing SR. These results parallel findings of earlier theoretical studies [5,9], which have considered the effect of network size on SR. It is worth noting that other than findings reported here, results from additional simulations indicated that in comparison to either only manipulating the values of a fixed $G$ or the ranges of the $G$ distributions but keeping the mean constant, the stochastic $G$ manipulation as implemented here yields the most unequivocal age differences in SR. Furthermore, consistent with earlier work on SR in multiunit summing network [5,9], increasing the number of units at the cortical layer (i.e., from $n_{2}=4$ to 50 ) extends the limit of sensitivity to optimal noise intensity for networks with all levels of $G$. The relative effect of $G$ on the magnitude of SR is, however, maintained: networks with smaller $G$ show lower peak SR. 

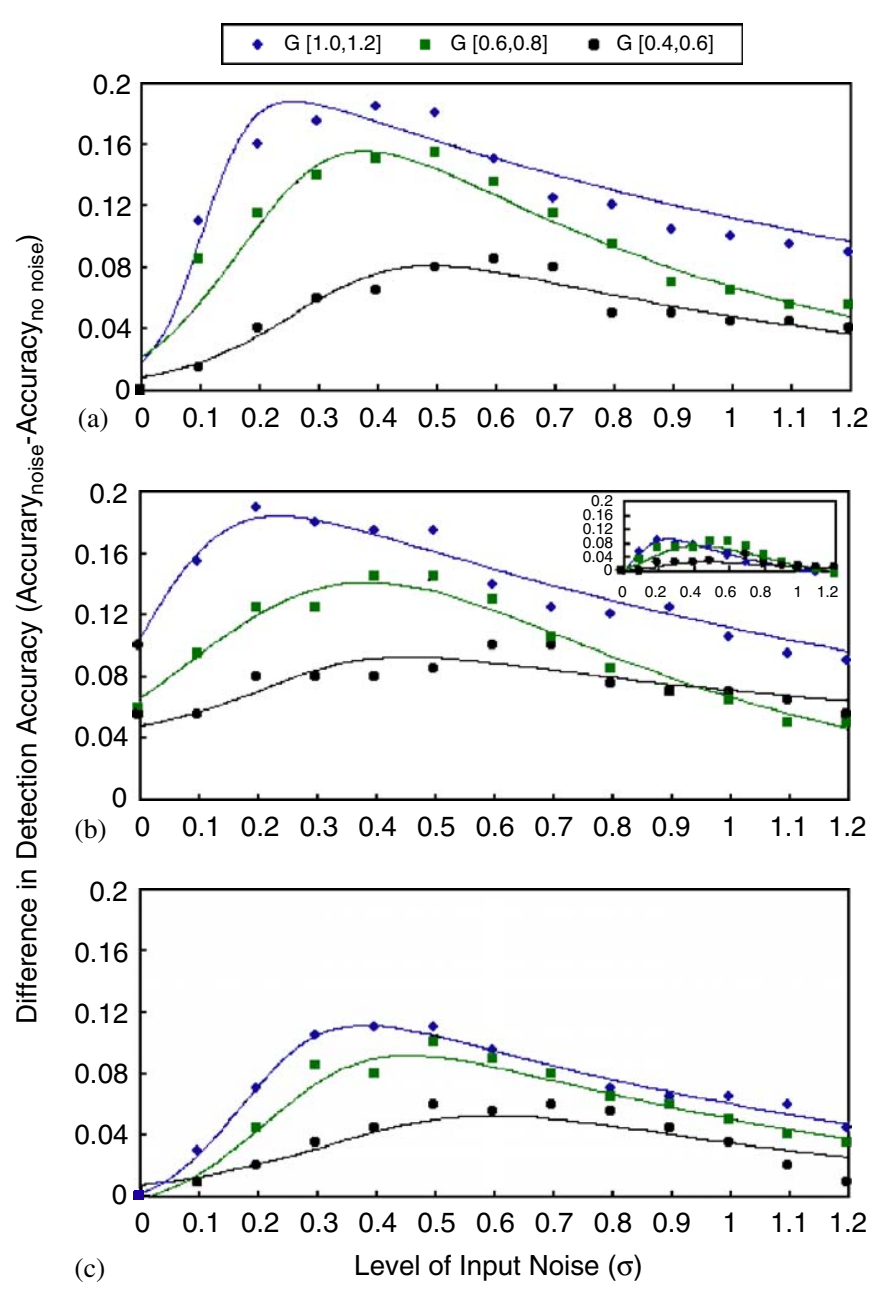

Fig. 4. Results of differences in proportion of detection accuracy between conditions with and without external noise as a function of external noise as simulated by the multi-unit, multi-layer networks. (a) SR functions with different levels of $G$. (b) SR functions assessed at the second testing session after exposure to subthreshold stimulus and external noise in a first testing session for different levels of $G$. (c) SR functions assessed after extensive exposure to superthreshold stimulus and external noise.

\subsection{Effects of repeated noise exposure}

The effects of short- and long-term repeated exposures to external noise were also investigated. The neurocognitive system is adaptive and plastic at various levels [31,40,41]. Gradual adaptations to external noise and weak signal may affect the SR effect. Hitherto, the effect of short-term exposure to external noise on SR in sensory detection had only been empirically examined in a small number of young subjects in up to two testing sessions. In the second session, only $50 \%$ of the subjects still showed an SR effect of a magnitude similar to the initial effect [7]. Analogous to the experimental procedure used in the empirical study, in one set of simulations we examined the effect of short-term repeated exposure to noise by comparing the SR effect assessed in a second testing session with that assessed in the first session. Following an initial training phase as described above, the connection weights of the networks were adaptively adjusted while being exposed to the subthreshold stimulus together with noise during the first test session. Simulation results presented in Fig. 4(b) show that absolute detection accuracy increases in the second testing session, as indicated by the greater-than-zero difference of detection accuracy at zero noise level. This effect is clearest in networks with the largest $G$, suggesting that networks with the largest $G$ learned most and became more familiarized with the weak signal. Second, although $\mathrm{SR}$ is still observed, the relative SR effects in comparison to those of the first session are reduced and this reduction is strongest in networks with the largest $G$ (see inserted panel, Fig. 4(b)).

The effect of long-term exposure to noise was examined in another set of simulations. After determining the optimal levels of external noise that produced the peaks of SR for networks associated with different levels of $G$, the networks were trained with 30 additional trials in which the original, superthreshold stimulus was embedded in the optimal level of external noise $(\delta=0.5$ in the simulations reported here). After 30 trials of noise exposure training, the networks were again tested with the subthreshold stimulus across different levels of external noise. Similarly, the simulation results show that gradual adaptations to long-term repeated exposures of external noise for over 30 sessions reduce the magnitude of SR (compare Fig. 4(c) with 4(a)). Again, this relative reduction is more pronounced in networks with larger $G$. Together, these results suggest that, when been repeatedly exposed to external noise, aging-related reduction in neural plasticity might protect old people from losing the SR benefit as drastically as young people, who pay the price of greater plasticity resulting in greater adaptation to subthreshold stimuli and repeated noise exposures.

\section{Discussion and conclusion}

Juxtaposing our findings of reduced and right-shifted peak SR with decreasing $G$ tuning and previous analytical models [5,9] showing similar effects with limiting the number of processing units suggests a conjecture: networks with attenuated $G$ function as if they have a more limited number of processing units. This sheds new light for understanding cognitive and sensory aging both at the neurochemical and neuroanatomical levels. Current evidence suggests that the effect of anatomical neuronal loss is small during normal relative to pathological aging. Milder cognitive and sensorimotor deficits occurring during normal aging are likely to be due to neurochemical shifts in relatively intact neural networks [28]. As conjectured here, without explicitly modeling anatomical neuronal loss, networks with an equal number of processing units but attenuated $G$ can be functionally similar to networks with fewer units.

Our simulation results here also corroborate previous findings on linearization of the input-output transfer function by external noise in the context of aperiodic SR 


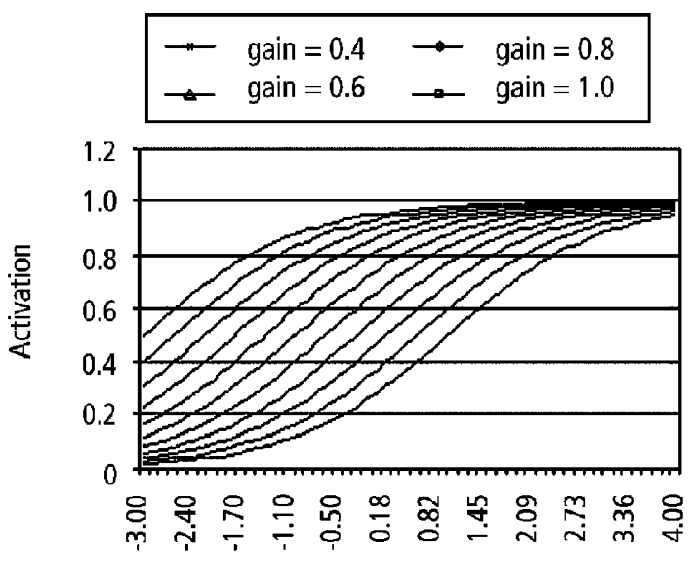

(a)

Signal
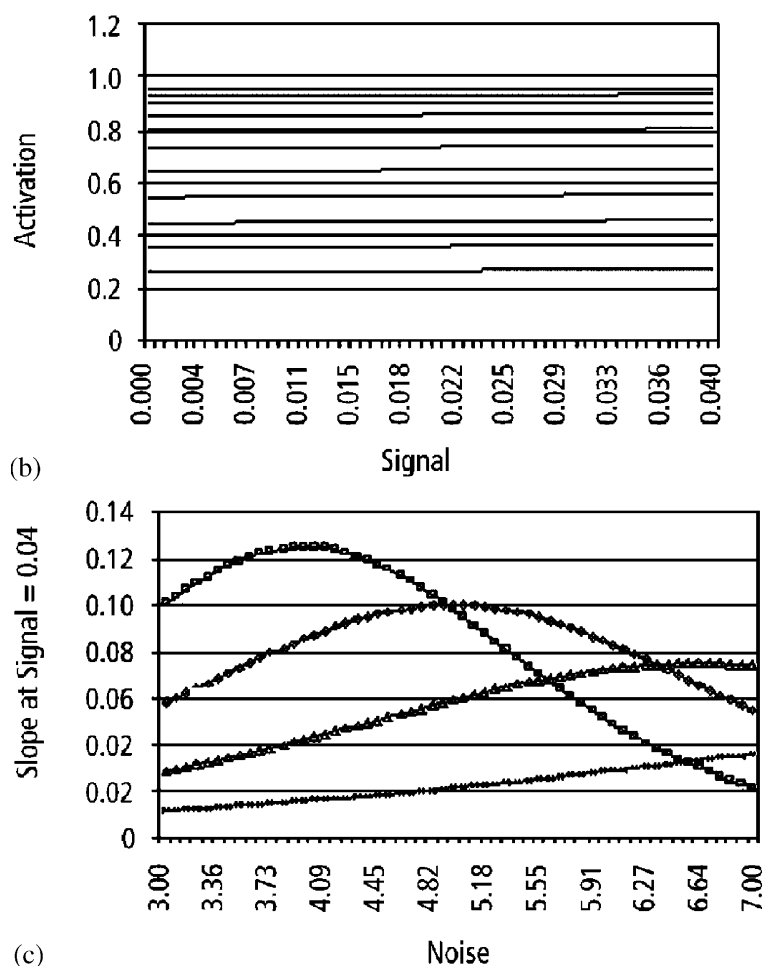

Fig. 5. Simulations results regarding interactions between system-parameter-based gain regulation and external-noise-induced tuning effect. Panel (a) depicts mean activation curves in the typical sense of input SNR. The $x$-axis gives the mean strength of the signal, and the $y$-axis gives the activation of a single unit. The different curves represent different levels of noise (3.0-7.0). Each curve is associated with a given level of noise and depicts the mean activation function across a range of signal strengths. Panel (b) displays data from (a), restricted to a tight interval of signal strength from 0.0 to 0.04 (see explanations in text). Panel (c) depicts slopes for different curves at a signal level of precisely 0.04 across the noise levels between 3.0 and 7.0. The curve for gain $=1.0$ corresponds directly to panels (a) and (b). To see the interactions between system gain regulation and external-noise-induced tuning, the other three curves plot the similar effect for networks with lower mean values of system gain (see text for further explanation).

[3]. In addition, we demonstrate how external-noiseinduced tuning interacts with the system's intrinsic gain regulation. As shown in Fig. 5(a), one way to illustrate the effect of external noise on signal detection (as was done, e.g., in [3]) is to plot the mean activation for a given signal strength across different noise levels (i.e., the input SNR function at different levels of external noise). Focusing on a very tight range of signals $(0.0-0.04)$, we see in Fig. 5(b) that the slopes (visualized here as more discrete rising steps in the curves) of the uppermost (corresponding to high external noise level) and the lowermost curves (corresponding to low external noise level) are less steep than the slopes of curves in the center (corresponding to optimal noise range for external-noise-based SR effect). In other words, at low and high levels of external noise the slope of the SNR transfer function is reduced, as shown in the previous study [3]. To visually illustrate the differences in slopes of the SNR transfer function more clearly as it was done in previous work [3], Fig. 5(c) depicts the slopes of the different curves at a signal level of precisely 0.04 across external noise levels from 3.0 to 7.0. Furthermore, Fig. 5(c) also displays a new additional aspect: the slopes of the SNR transfer function are affected by different levels of intrinsic system gain regulation. The curve for gain $=1.0$ corresponds to the plots in panels a and b of Fig. 5. The other three curves represent the slopes of the SNR transfer function for systems with three lower levels of intrinsic system gain regulation. It is clearly seen that the effect of external noise tuning interacts with the level of the system's internal gain regulation. In line with the simulation results reported in Figs. 2 and 4, analyzing the effect of system gain regulation on external-noise-induced SR in terms of the slopes of the SNR transfer function also shows that systems with suboptimal intrinsic gain regulation (i.e., lower values for the gain parameters of their activation functions that simulate older people with less efficient neuromodulation) exhibit weaker and right-shifted SR effects.

Direct empirical studies on age differences in SR are scarce, as most studies examined SR in old [25] and young $[6,7]$ people separately. Our results point to a methodological issue regarding interpretations of age differences of SR in psychophysical experiments. Due to reduced sensory threshold sensitivity [24], a higher level of externally applied noise is usually required for old rather than young people [32]. Such age differential adjustments, however, set the to-be-observed empirical young and old effects on different parts of the SR functions, and hence render unambiguous comparisons of age effects difficult. Comparisons of the full psychophysical SR function using experimental paradigms with systematic parametric variations across external noise and stimulus levels in different age groups may more comprehensively capture age differences of SR.

In conclusion, the analytical and computational analyses reported here provide a framework for relating agingrelated neurobiological declines with the phenomenon of $\mathrm{SR}$. As processes of brain aging occur at neurochemical and neuroanatomical levels, increased neuronal noise and reduced neural plasticity may modulate the extent of the external-noise-enhanced SR effect and its habituation process. Our model suggests that as the aging brain 
becomes noisier internally, sensory aging is associated with reduced peak SR, higher level of external noise required for inducing SR, ${ }^{1}$ increased sensitivity to the range of optimal noise intensity, and decreased sensitivity to repeated noise exposure. We hope these predictions stimulate concerted empirical efforts for research on aging and SR.

\section{References}

[1] L. Bäckman, et al., Age-related cognitive deficits mediated by changes in the striatal dopamine system, Am. J. Psychiatry 157 (2000) 635-637.

[2] S.A. Castner, P.S. Goldman-Rakic, Enhancement of working memory in aged monkeys by a sensitizing regimen of dopamine D1 receptor stimulation, J. Neurosci. (2004) 1446-1450.

[3] D.R. Chialvo, A. Longtin, J. Müller-Gerking, Stochastic resonance in models of neuronal ensembles, Phys. Rev. E. 55 (1997) 1798-1808.

[4] S.J. Colcombe, et al., Aerobic fitness reduces brain tissue loss in aging humans, J. Gerontol. 58A (2003) M176-M180.

[5] J.J. Collins, C.C. Chow, T.T. Imhoff, Stochastic resonance without tuning, Nature 376 (1995) 236-238.

[6] J.J. Collins, T.T. Imhoff, P. Grigg, Noise-enhanced tactile sensation, Nature 383 (1996) 770.

[7] J.J. Collins, T.T. Imhoff, P. Grigg, Noise-mediated enhancements and decrements in human tactile sensation, Phys. Rev. E. 56 (1997) 923-926.

[8] J.M. Fellous, C. Linster, Computational models of neuromodulation, Neural Comput. 10 (1998) 771-805.

[9] P.C. Gailey, et al., Stochastic resonance in ensembles of nondynamical elements, Phys. Rev. Lett. 79 (1997) 4701-4704.

[10] L. Gammaitonim, et al., Stochastic resonance, Rev. Mod. Phys. 70 (1998) 224-287.

[11] C.L. Grady, A.R. McIntosh, F.I.M. Craik, Age-related differences in the functional connectivity of the hippocampus during memory encoding, Hippocampus 13 (2003) 572-586.

[12] D.C. Gravelle, et al., Noise-enhanced balance control in older adults, Neuroreport 13 (2002) 1853-1856.

[13] M.E. Hasselmo, Neuromodulation: acetylcholine and memory consolidation, Trends Cognitive Sci. 3 (1999) 351-359.

[14] C. Ivey, A.V. Apkarian, D.R. Chialvo, Noise-induced tuning curve changes in mechanoreceptors, J. Neurophysiol. 79 (1998) 1879-1890.

[15] V. Kaasinen, et al., Aging-related dopamine D2/D3 receptor loss in extrastriatal regions of the human brain, Neurobiol. Aging 31 (2000) 683-688.

[16] C.F.A. Kugler, A. Taghavy, D. Platt, The event-related P300 potential analysis of cognitive human brain aging: a review, Gerontology 39 (1993) 280-303.

[17] H. Lecar, R. Nossal, Theory of threshold fluctuations in nerves. II. Analysis of various sources of membrane noise, Biophys. J. 11 (1971) 1048-1067.

[18] S.-C. Li, U. Lindenberger, P.A. Frensch, Unifying cognitive aging: from neuromodulation to representation to cognition, Neurocomputing 32-33 (2000) 879-890.

[19] S.-C. Li, U. Lindenberger, S. Sikström, Aging cognition: from neuromodulation to representation, Trends Cognitive Sci. 5 (2001) 479-486.

[20] S.-C. Li, M. Naveh-Benjamin, U. Lindenberger, Aging neuromodulation impairs associative binding: a neurocomputational account, Psychological Science 16 (2005) 445-450.

\footnotetext{
${ }^{1}$ After submission of this paper, the intriguing theoretical prediction of older, less well neuromodulated neurobiological systems requiring more external noise to benefit from stochastic resonance has recently been independently confirmed in an empirical study on aging and vibrotactile sensitivity by Wells et al. [43].
}

[21] S.-C. Li, S. Sikström, Integrative neurocomputational perspectives on cognitive aging, neuromodulation, and representation, Neurosci. Biobehav. Rev. 26 (2002) 795-808.

[22] S.-C. Li, et al., Short-term fluctuations in elderly people's sensorimotor functioning predict text and spatial memory performance: the MacArthur Successful Aging Studies, Gerontology 47 (2001) $100-116$.

[23] S.-C. Li, et al., Transformations in the couplings among intellectual abilities and constituent cognitive processes across the life span, Psychological Science 15 (2004) 155-163.

[24] U. Lindenberger, P.B. Baltes, Sensory functioning and intelligence in old age: a strong connection, Psychol. Aging 9 (1994) 339-355.

[25] W. Liu, et al., Noise-enhanced vibrotactile sensitivity in older adults, patients with stroke, and patients with diabetic neuropathy, Arch. Phys. Med. Rehabil. 83 (2002) 171-176.

[26] Z.L. Lu, B.A. Dosher, External noise distinguishes attention mechanism, Vision Res. 38 (1998) 1183-1198.

[27] S.W.S. MacDonald, D.F. Hultsch, R.A. Dixon, Performance variability is related to change in cognition: evidence from Victoria longitudinal study, Psychol. Aging 18 (2003) 510-523.

[28] J.H. Morrison, R.P. Hof, Life and death of neurons in the aging brain, Science 278 (1997) 412-429.

[29] F. Moss, L.M. Ward, W.G. Sannita, Stochastic resonance and sensory information processing: a tutorial and review of applications, Clin. Neurophysiol. 115 (2004) 267-281.

[30] N.A. Nessler, A.M. Shirke, R. Malinow, The probability of transmitter release at a mammalian central synapse, Nature 366 (1993) 569-572.

[31] L. Nyberg, et al., Neural correlations of training-related memory improvement in adulthood and aging, Proc. Natl. Acad. Sci. USA 100 (2003) 13728-13733.

[32] A. Priplata, et al., Vibrating insole and balance control in elderly people, Lancet 362 (2003) 1123-1124.

[33] N. Raz, U. Lindenberger, K.M. Rodrigue, et al., Regional brain changes in aging healthy adults: general trends and individual differences, and modifiers, Cerebral Cortex 10 (2005).

[34] A.A. Saha, G.V. Anand, Design of detectors based on stochastic resonance, Signal Process. 83 (2003) 1193-1212.

[35] E.L. Schneider, Handbook of the Biology of Aging, Academic Press, New York, 1996.

[36] G. Segovia, et al., Glutamatergic neurotransmission in aging: a critical perspective, Mech. Ageing Dev. 122 (2001) 1-29.

[37] D. Servan-Schreiber, H. Printz, J.D. Cohen, A network model of catecholamines effects: gain, signal-to-noise ration and behavior, Science 249 (1990) 892-895.

[38] E. Simonotto, et al., Visual perception of stochastic resonance, Phys. Rev. Lett. 78 (1997) 1186-1189.

[39] E. Simonotto, et al., FMRI studies of visual cortical activity during noise stimulation, Neurocomputing 26-27 (1999) 511-516.

[40] W. Singer, Development and plasticity of cortical processing architecture, Science 270 (1995) 758-764.

[41] T. Singer, U. Lindenberger, P.B. Baltes, Plasticity for new learning in very old age: a story of major loss?, Psychol. Aging 18 (2003) 306-317.

[42] A.T. Welford, Signal, noise, performance, and age, Hum. Factors 23 (1981) 97-109.

[43] C. Wells, et al., Touch noise increases vibrotactile sensitivity in old and young, Psychol. Sci. 16 (2005) 313-320.

[44] K. Wiesenfeld, F. Moss, Stochastic resonance and the benefit of noise: from ice ages to crayfish and squids, Nature 373 (1995) 33-36.

[45] D.F. Wong, et al., Effects of age and dopamine and serotonin receptors measured by positron tomography in the living human brain, Science 226 (1984) 1393-1396.

[46] B. Xu, F. Duan, F. Chapeau-Blondeau, Comparison of aperiodic stochastic resonance in a bistable system realized by adding noise and by tunning system parameters, Phys. Rev. E 69 (2004) 061110-1061110-8. 


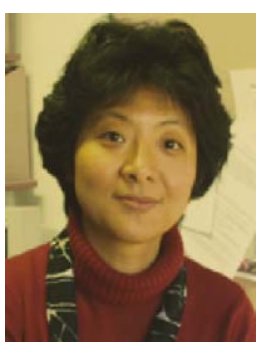

Shu-Chen $\mathbf{L i}$ is a research scientist in the Center for Lifespan Psychology at the Max Planck Institute for Human Development. Her research interests include behavioral studies of lifespan cognitive and sensorimotor development, and cognitive and computational neuroscience. Theoretically, she is particularly interested in modeling behavioral phenomena of lifespan development by computationally implementing neurobiological mechanisms that have bearings on memory, binding, attention, and processing speed.

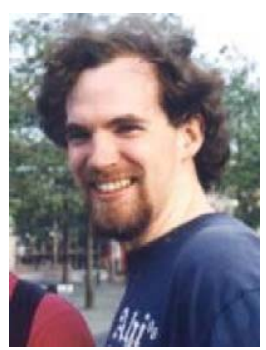

Timo von Oertzen is an assistant professor in the Department of Mathematics at Saarland University, Saarbrücken, Germany. He is a computer scientist by training, with research interests in computational mathematics and computational psychology. He has also worked on algorithms for symbolic number representation and automated proving.

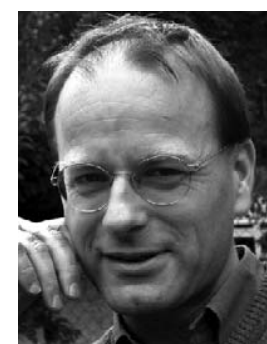

Ulman Lindenberger is a professor of psychology and directs the Center for Lifespan Psychology at the Max Planck Institute for Human Development. He studies cognitive and sensorimotor development across the lifespan. Specifically, he is interested in mechanisms regulating links between domains of functioning (e.g., sensorimotor, cognitive) and levels of analysis (e.g., behavioral, neuronal) during learning and lifespan development. 